\title{
Supporting peace of mind and independent living with the Aurama awareness system
}

\author{
Pavan Dadlani ${ }^{\mathrm{a},}{ }^{,}$, Panos Markopoulos ${ }^{\mathrm{b}}$, Alexander Sinitsyn ${ }^{\mathrm{a}}$ and Emile Aarts ${ }^{\mathrm{a}, \mathrm{b}}$ \\ ${ }^{a}$ Philips Research, High Tech Campus 34, 5656 AE, Eindhoven, The Netherlands \\ ${ }^{\mathrm{b}}$ Department of Industrial Design, Eindhoven University of Technology, P.O. Box 513, Den Dolech 2, Eindhoven, \\ $5600 \mathrm{MB}$, The Netherlands
}

\begin{abstract}
An awareness system was designed to provide peace of mind and a sense of connectedness to adults who care for an elderly parent living alone. The iterative design of the Aurama awareness system showed and our empirical research, including field trials ranging from four to six months, confirm the potential of awareness systems to support both generations suggesting that research should examine, firstly, how to convey long-term trends regarding the wellbeing of the elderly and, secondly, how to intertwine the communication of awareness information with expressive forms of communication. We further explore the role that formal care providers can play when caring for the elderly using the awareness system. We discuss implications of our studies for the design of ambient intelligent systems supporting awareness between elderly, their adult children and care providers, and derive several lessons learned in conducting long-term field trials.
\end{abstract}

Keywords: Ambient intelligence, awareness, connectedness, assisted living, field trials, elderly care

\section{Introduction}

The elderly population is expected to grow rapidly in developed countries in the next 50 years. Extrapolating current trends, the older population in 2030 in the US is expected to be twice as large as in 2000, growing from 35 million to 71.5 million and representing nearly 20 percent of the total population [1]. In many other developed regions like Europe, the proportion of seniors to the rest of the population is expected to become even greater [21]. These trends inflate the so-called 'sandwich' generation: adults with children and an aging parent to take care of. As a consequence it is expected that the children of these elderly will need to take an important role in caring for the elderly's wellbeing. This research explores the potential of using Ambient Intelligence technology (AmI) $[4,9]$ as part of this care process; specifically we explore how technology that supports awareness regarding an elder's activities and whereabouts can offer peace of mind to both the elder and the adults who are peripherally involved in the care of their parents. Awareness also helps both sides ad- dress their need for a sense of connectedness, understood here as a positive emotional experience, characterized by a feeling of staying in touch [6] within ongoing social and caring relationships.

The class of AmI systems that this research is concerned with is often referred to as 'awareness systems' to indicate that their primary purpose is to create awareness of another's activities or whereabouts, and an understanding of their status and context. This understanding provides a backdrop for shared activities and social interactions [27]. This purpose can be served by non real-time and non rich media, in contrast to traditional communication systems' goals such as efficient and effective information exchange between users, or providing a sensation of being close together while apart, a vision that has dominated much of telecommunication research relating to the concept of social presence [19].

Several research studies examine the scenario of monitoring a remote elder; most notably CareNet [1], Digital Family Portrait (DFP) [3,11] and Diarist [2]. Such studies provide converging evidence regarding the potential of awareness systems, understood here

*Corresponding author. E-mail: pavan.dadlani@philips.com. 
as systems that help connected individuals stay informed regarding the activities and status of each by providing frequent and partly automated updates. There have been some deployments of experimental awareness systems supporting the scenario of monitoring a remote elder but they are scarce, they tend to be small scale, and the evidence they report regarding their benefits is still very tentative. For example, CareNet focused on providing visualizations of information regarding the wellbeing and activities of daily living of an elder to a carer who provides care for the elder on a frequent basis (defined by them as 'drastic life changers' or 'significant contributors', as opposed to those who are 'peripherally involved' [1]). Although they suggest that such systems can improve the quality of care for an elder, their evaluation was only based on Wizard of $\mathrm{Oz}$ methods, leaving unexplored the sensor infrastructure and its acceptance in real situations. On the other hand, the DFP deployed a sensor infrastructure and focused on presenting awareness of the elder's general activity level, blending this information aesthetically and blending in the home environment like a picture frame. Their single deployment was only for 6 weeks and showed the benefits of the awareness system to provide peace of mind and tackle loneliness of elderly. Similarly, Diarist provided peace of mind, connectedness and social presence. Furthermore, it provided information in different levels: from high-level awareness to detailed narratives of the elder's routine. However, their system was deployed twice and for a period of one week only.

Importantly, driven by the aim to bridge distance, earlier works have focused on supporting awareness at a relatively fine time-scale: moment-to-moment presence, or activities of daily living. This seems to be the obvious target, firstly because of the relative technological ease of detecting these activities, and secondly because of the traditional aspiration to emulate physical proximity: systems are aiming to substitute some of the awareness that individuals could have of each other if they were collocated. In this work we argue that such an approach is not necessarily desirable; we commence our investigation by trying to understand the awareness needs for both parties and follow on to propose relevant design solutions.

In addition to research prototypes, there are also several products and services commercially available that allow care providers and family members to be informed about the status of an elderly. Some examples are QuietCare [12], HealthSense [13] and WellAware [14]. These systems are typically imple- mented in terms of three components: motion sensing, a base station, and a web-based service by which carers can obtain detailed information about the elder's wellbeing. They generate information on the activities of daily living (ADLs) of the elder (e.g., cooking, toilet use, etc.). However, these systems provide usually a one-way flow of information and focus purely on monitoring the elderly looking for abnormalities. In both research and practice, the systems are typically one-way flow of communication and monitoring, and even though the elderly are kept at the center of attention to support their independent living, very little thought has been put into social connectedness, and getting elderly to accepting these services.

Taking a step back, this research questions what are the needs for awareness between seniors and their adult children, and what elements are required for these technologies to be adopted. Compared to those earlier works, more emphasis is also put on the hardware design and its aesthetics, which are often argued to be important in this field, see for example $[3,11]$.

Section 2 summarizes the design and initial evaluation of the Aurama awareness system, which led to its redesign reported in Section 3. Section 4 describes the long-term field trials conducted, involving nine participants for a period of four to six months. Next, based on the findings from deploying the system and conducting a study with care organizations, a third generation of the Aurama awareness system was designed and implemented. We end with several implications for the design of ambient assisted living systems, lessons from conducting longterm field trials, and conclusions from this work.

\section{Designing Aurama}

\subsection{Initial focus groups and interviews}

Four focus groups $(\mathrm{N}=19)$, interviews with caregivers $(\mathrm{N}=8)$, and interviews with seniors $(\mathrm{N}=7)$ were held. Participants were different and not related in each of these studies. The aim for this initial study was to get a feel for the current situation between elderly and their adult children and capture their needs for awareness information. These sessions challenged several of the assumptions that are taken for granted in some of the works reviewed above. Thus, the goals of the study were: (1) to evaluate the concept of providing awareness information to both 

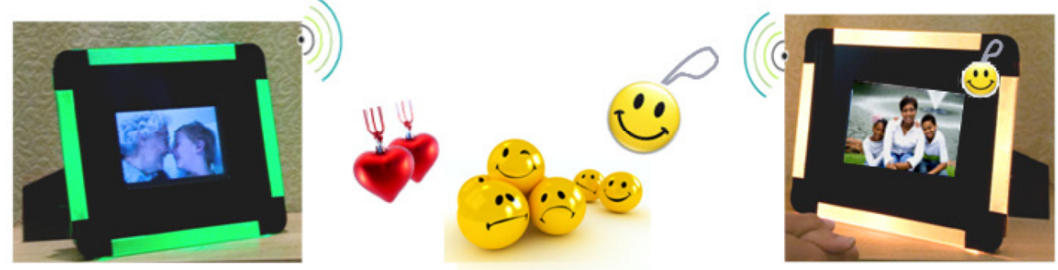

Fig. 1. Intelligent photo frame jewelry provides explicit awareness of affections.

parties; (2) to find out what should the information consist of; and (3) evaluate concepts of the awareness system. While the detailed results of this study can be found in [7], a summary of the findings is provided below.

Rather than detailed trivia of daily activities, or real time updates regarding whereabouts, care giving children wish to obtain high-level overviews about their parents' wellbeing. Rather than a constant feed of information, they wish to be informed regarding changes to known daily life patterns, and of gradual shifts in lifestyle that may not be easy to notice during visits or direct communication. In agreement with the thesis of Hollan and Stornetta [20], they did not look for emulating the awareness one obtains from face to face interaction, but to complement it. Adult children value the possibility to see historical data, but they found the idea of receiving daily status reports or a 'story' with detailed schedules and activities too invasive.

Privacy and burden have dual connotations: on one hand, elderly do no mind sharing their wellbeing information with their children, but fear of putting a burden on them; on the other hand, (adult) children want to be more involved in their parent's wellbeing by knowing more about changes of long-term patterns, but they fear of invading the privacy of their elderly parent.

Regarding the presentation of the awareness information, participants favored ambient displays such as interactive digital photo frames, over direct channels such as SMS and e-mail. By ambient display we mean a decorative and always-on display, that participants can ignore safely most of the time, but which they can turn their attention to when they wish to. It is particularly important for these displays to not be disruptive demanding the attention of the user to handle notifications and respond to updates and messages provided by the system [18].

\subsection{The first Aurama prototype}

To verify the reported preference of long-term trend awareness, we designed and implemented a system supporting awareness of presence at home, sleep patterns, weight variations, and cognitive ability regarding the lone elder. The system includes an interactive photo frame which emits colored light 'auras' presenting at-a-glance information regarding the elder (see Fig. 1). If the elder is at home, a blue aura appears on the child's frame, which disappears if the elder leaves. To provide awareness of deviations from known patterns of behavior that the system detects, a yellow or orange aura is shown depending on the magnitude of this change or deviation. The frame displays charts showing long-term trends of the elder's data.

Presence at home is detected via a wireless tag transmitting RF signals and placed on a keychain carried by the elder. Weight is measured with load sensors placed under each leg of the bed. The same load sensors are used to monitor the number of times the elder gets up during the night and the amount of sleep the elder gets. This information is used to give an estimation of their sleep quality. Clearly, how many times one gets up in the night is but a weak approximation of what might be considered as an appropriate measure of sleep quality. This approximation though was easy to build and could help participants to experience first hand what remote monitoring of sleep quality would feel like for both parties, the 'watcher' and the 'watched'. Data about sleep and weight was assessed once a day to identify unusual events. Cognitive ability, however, was reassessed every time the elder finished playing some computer games that address different cognitive skills. This was possible via the elder's photo frame which was also the window to their own data, i.e., similar visualizations the child had access to. 


\subsection{Laboratory evaluations and short field trials}

Participants $(\mathrm{N}=6)$ in lab-based trials were invited to test the usability of the system, e.g., test the interpretation of the visualizations and colored auras on the picture frame, and obtain an overall impression of the application. We then conducted two field trials of two weeks each $(\mathrm{N}=6)$, to test the experience of the awareness system under realistic conditions, evaluate privacy and acceptance, while focusing on its added value to bring elderly and their children closer. All participants were different than those in the original inquiries. Details and methodologies of these studies can be found in $[7,10]$.

These studies revealed that participants appreciate the concept of the Aurama system to capture changes in long-term trends and were assured that the system will help them access wellbeing information easily and help them be more involved in their parents' wellbeing. Users appreciated the design aesthetics of the display and valued the colored auras to indicate the status information of a close relative. Users valued the emphasis on coarse grain information, confirming our choice to deviate from earlier designs, as for instance [2]. Participants expressed a major interest for the positive aspects in their parents' lives to further foster peace of mind rather than only the negative events that the system focused upon. Furthermore, many were keen about the mood and emotional feelings of their parent, which they find difficult to assess with phone calls and visits. Often the adult children fear being intrusive, even though their parents claim that they do not mind sharing the information, corroborating our findings from initial studies.

In contrast to earlier studies [1-3,11], our initial trials further emphasized the need to know about changes in wellbeing that are difficult to assess during visits and phone calls, such as long-term trends, moods, and the general reassurance that things are going as anticipated; the latter being in accordance with earlier studies $[16,17]$.

In one of the trials, the elder did not take the presence tag with him when leaving out of home, and erroneous presence information was shown to the child. This led to a false alarm when nobody picked up the phone calls the child was making, showing that trust and reliability is crucial for the acceptance of such systems. Furthermore, the short trials showed that at times the system was perceived as a monitoring system, especially for elderly, since it was mainly one-way flow of information. In fact, at the end of the trials the elderly stated they would like to receive awareness information regarding the status of their children and their family (e.g., grandchildren).

\section{Second generation of the awareness system}

Based on lessons learnt from the first field trials, several changes were made to the awareness system. We wanted the Aurama system to move away from being a clinical 'monitoring' system that one remembers to use only for checking the wellbeing of the elderly simply out of duty. For this, two things were required: firstly, making the system more symmetric and secondly, de-emphasizing the wellbeing information while making Aurama a venue for regular playful and pleasant interaction that makes them feel connected. Thus, checking long-term trends of the elder becomes a secondary use but still a natural one. Additionally, we want to allow the expression of positive messages and provide a glimpse to the emotional feelings of the elder. To meet these requirements, we expanded the Aurama system to support affective messaging via the picture frame by means of intelligent photo frame jewelry (Fig. 1). This allows users to express emotions explicitly in a playful manner: placing a tagged object (smiley, love or cry) on the picture frame creates a colored aura on the frame at the remote site, together with a graphical representation of the emotion. To further reduce or eliminate asymmetry, the revised design allows presence information to also be bidirectional, so both parties' key chains are augmented to share presence awareness of each other.

Furthermore, it was also noted that the field trials were too short to provide useful trend information on cognitive abilities, so the cognitive games were removed from the system.

Figure 2 shows the system's architecture and examples of long-term trends. To further examine the interest for users of long-term trend information, in addition to sleep and weight charts, we developed an overview of presence at home over time, which facilitates the detection of abnormal patterns and changes in habits. Figure 3 shows a sample chart for presence patterns.

\section{Long-term field trials}

We were aware that the initial trials in the field were too short to observe anything resembling a long-term trend. We needed participants to have a 


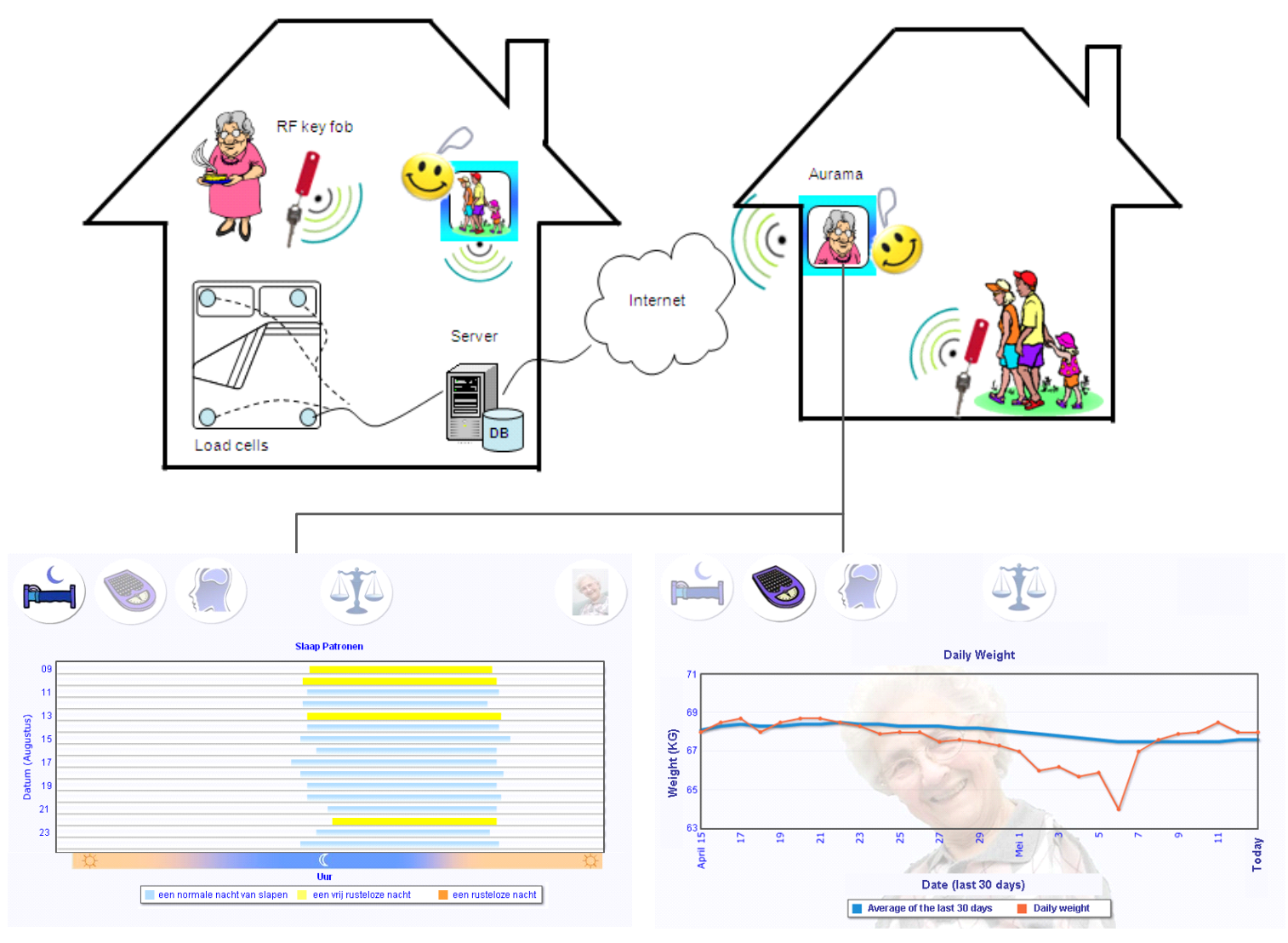

Fig. 2. System's architecture and sample charts for sleep and weight patterns.

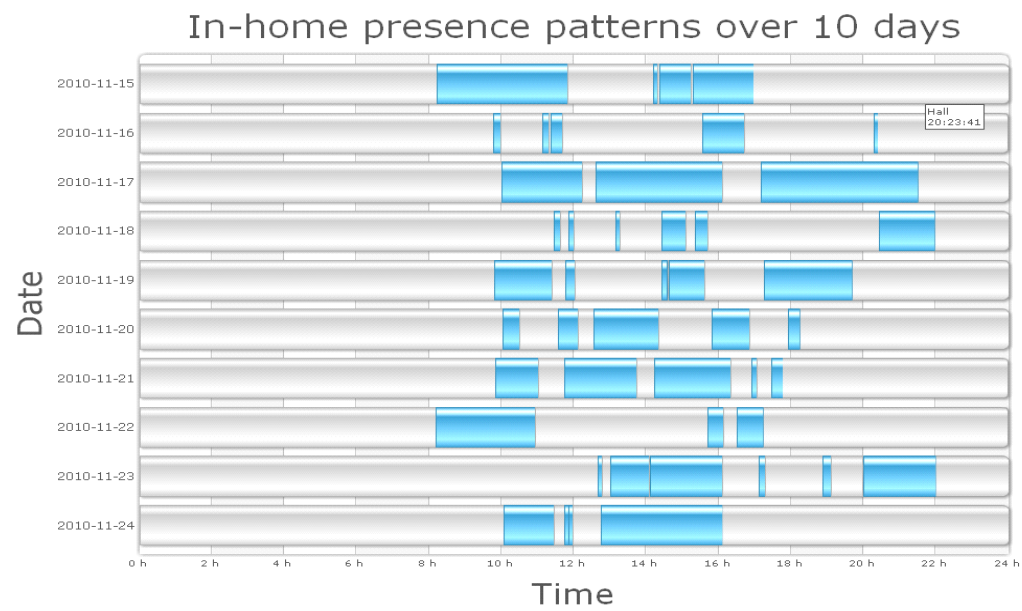

Fig. 3. Sample chart displaying presence patterns of the elder showing when the elder is in (lighter timeslots) or out (darker timeslots). 
more realistic experience of the Aurama system. Especially we wanted to see if the system is used or is gradually abandoned after a few days, if it becomes an integral part of the communication between parent and child, or something that they do just for the experimenter's sake or for reasons relating to study setup rather than to support their actual communication needs. To evaluate the revised system, Aurama was deployed for two longer trials: one lasting for six months and the second one for four months. In total nine subjects participated in this study. The first trial involved an 85-year old woman living alone in a home care community and at a distance $(70 \mathrm{~km})$ from her son, his wife and her two granddaughters. The second one involved an 82-year old woman also living in a home care community about $135 \mathrm{~km}$ from her son, his wife and her grandchild.

The trials consisted of three phases: (1) the sensor infrastructure was deployed and was used to collect data for a period of time, (2) the ambient display was installed on both locations where awareness of presence and wellbeing was provided, and (3) the set-up was enhanced with explicit exchange of affective messages. Similar to the initial short trials, interviews and questionnaires were conducted before installing the system and after each phase. However, since the results of the questionnaires alone in the initial trials did not provide convincing results as the qualitative feedback did, we used the questionnaires measuring connectedness [23], the affective benefits and costs of the awareness system [24], and the usefulness of the system [25] to design a semi-structure interview based on several items of those questionnaires. Topics included obligations and expectations felt during the use of the system, privacy concerns, shared experiences, feelings of closeness, and usage patterns. Each interview was audio recorded lasting about half-hour and was conducted individually.

The data collection in phase 1 of the first longterm trial lasted for three months, while the second trial's phase 1 lasted for two months. The set-up took longer than expected since it was important to make sure that the technology was working properly to avoid going into the participants' home multiple times during the following phases, as we experienced during the initial short trials. It was also important to collect enough data to create a baseline and define thresholds for patterns, and configure the technology for that particular household.

Although the field study was not controlled, there were several expectations based on the study set-up. It had been expected that at the beginning of the deployment period the communication patterns between parent and child would have not yet been influenced, but the novelty effect of the sensor technology at home would have worn off by then and the technology would be less disruptive than when it was first introduced. This is another advantage for having a long phase 1 for configuring the technology and collecting data. Furthermore, we also expected some privacy concerns to arise already, mostly related to the installation of the sensor technology to monitor their daily activity.

In the second phase of the trial we expected to be able to evaluate what the effect is of having the awareness display available and how that influences communication patterns and feelings. We expected stronger feelings of connectedness and that both parties would be more reassured. We did worry about privacy concerns to arise, relating also to the disclosure of information about their private life and their status to their children. We expected also children to express concerns about being offered a glimpse to private aspects of their parent's life - this especially following earlier research (e.g., Diarist where such concerns were expressed from the part of the children and the initial interviews and focus groups). Finally, we expected that the introduction of emoticon-based communication would increase usage of the device, would make it be perceived as more playful and personal, and would reduce privacy concerns, since the whole 'monitoring' component of the application is embedded inside the context of a regular communication activity.

\subsection{First long-term field trial}

During the second phase, the system could not be used for three weeks due to technical problems. While the system worked in this phase, the grandchildren and daughter in law checked every 2-3 days every wellbeing parameter presented in the frame. The son, however, would not check that often directly, but preferred to learn about it through his family, preferring them to act as intermediaries to get the information. Figure 4 shows the usage patterns of the child and his family with the Aurama system.

The elder did not sleep well for several nights throughout the study and as a result yellow auras appeared on the child's frame. The family called to inquire if she had a bad night of sleep and felt 'obliged' to react, but they considered that an act of care and a responsibility they want to have, instead of a burden. In fact, they appreciate receiving such 'negative' news because they can get more involved in the elder's wellbeing. Further corroborating with 
Family's interaction with the ambient display

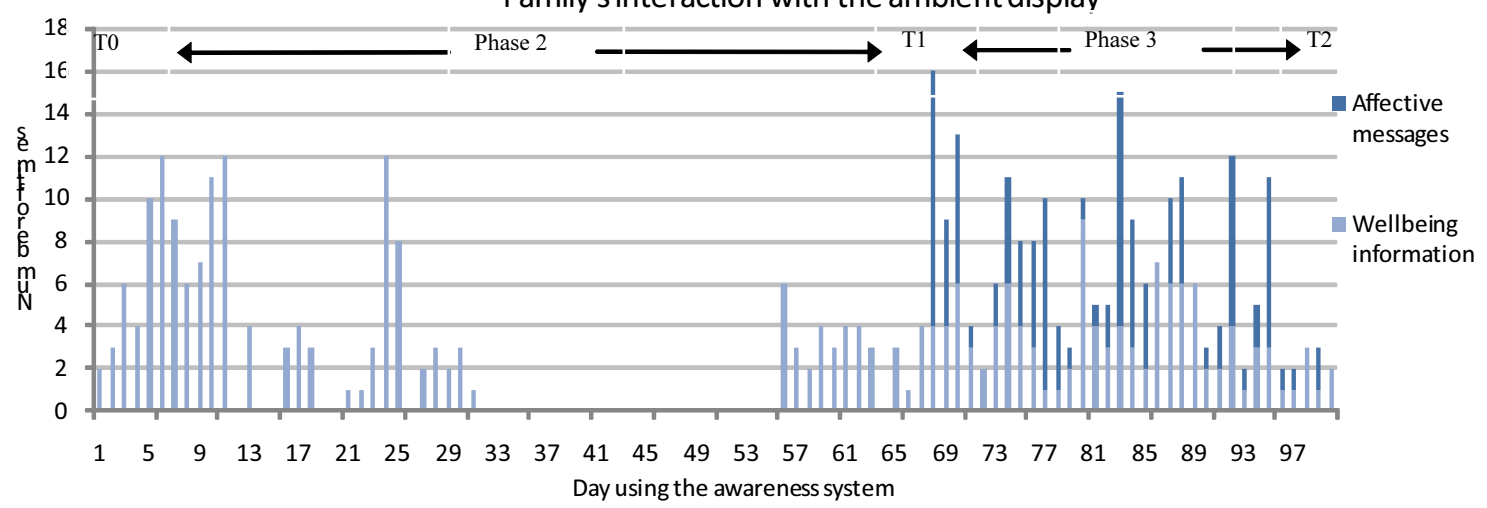

Fig. 4. Sample usage patterns for the remote family in the second and third phase of the study of the first long-term field trial.

our preliminary field trials [7], they valued seeing wellbeing information that they have no access to otherwise; especially non-detailed long-term patterns gave them rich information to assess wellbeing.

The experiences of the five participants were surveyed at three times, $T_{0}, T_{1}$, and $T_{2}$ (see Fig. 4). At $T_{1}$ they reported feeling that the system is not necessary yet because the elderly is still healthy, active and can call if there is a problem. Although the elder does not mind sharing the information with them and finds it pleasant to get calls when she does not sleep well, she feels she has enough contact and that it would be more useful for frailer elderly or be more relevant for her if she would not be living in a care facility. They do see the value, nevertheless, as a way to assess gradual decline.

At $\mathrm{T}_{2}$, participants reported that they enjoyed communicating emotions by means of the 'jewelry' and the auras. They exchanged emotions multiple times a day. Love and smiley were used the most. Although they considered that presence and wellbeing awareness made them think more about each other, they reported feeling more connected during the third phase. Several times, an exchange would trigger a phone call. The granddaughters, for example, confirmed that the number of contacts increased during the third phase.

The exchange of affective messages was also used as a reassurance mechanism. For example, the family learnt to expect every morning affective auras from the elder. A few times when this was missed from the elder, the family would take the initiative of sending her an emotion, and she would reply back. The elder also felt the same: 'If you see the smiley aura then you know that everything is fine'. If they receive an emotion, they felt they had to reply and if they sent one, they would expect a reply. Although this feature created a level of obligation and expectation, they found it enjoyable and useful.

Participants were satisfied with the three emotions and thought that more would confuse the elder. There is, however, a need for more meaningful and short messages, e.g., good morning, hello!, and the ability to talk through the ambient display. The physical objects were preferred over screen-based emotions because the tangibility of the interaction was considered more fun and natural.

\subsection{Second long-term field trial}

At $T_{1}$ we found that the child enjoyed the presence awareness which made him think more about his mother and feel more connected. Having the blue aura was a pleasant experience for him too. However, one of his home guests commented that 'you live with your mother again because she can see when you are home and when you are out'. Also, since the blue aura was quite salient, his neighbors could see whether his mother was 'in or out'. Although these comments did not bother him, it made him realize that his mother is 'there' and started to fear that she could call and ask 'where did you go, you were not at home'. This happened once and the daughter in law was not pleased with that.

At the beginning there was quite some interaction with Aurama due to its novelty in the child's environment. After sometime however, they would only check if something was wrong or has changed. The family valued highly being notified about changes in 
patterns such as a couple of bad nights of sleep causing an orange aura to be formed on Aurama. This made them give a call not just after being informed but around those days as well to check if she had recovered. If there were no changes, the child would check only once a week or so. He did not feel he was intruding in her life but rather felt more involved in her wellbeing since they were not in the habit of communicating as much as his brother would with her. In fact, most of the wellbeing information on his mother he would typically get through his brother. But he valued that he can get a direct glimpse to his mother's wellbeing.

The picture frame was once again perceived as too bulky and due to the hidden PC there was some noise caused by the PC's fan. Furthermore, the user interface was not easy to read for the child and would have liked more clear and detailed user interfaces.

The child would also like to be informed about his mother's eating patterns, activity levels and medication intake. However, the child and his family would want a combination of direct and explicit communication with the awareness information provided by Aurama. Similar to previous trials, they would like to see and talk with the elderly through the picture frame. That explicit form of communication would be valued more than the affective messages experienced during the last phase. The emotions were used mostly at the beginning of phase 3 and although they thought it was fun they prefer to have more meaningful messages.

For the elderly, we came to know through the child that it was not entirely clear for her what the purpose of the system was. Although all received a similar training at the beginning when deploying the system, it appeared that the elderly did not grasp the concept or may have forgotten, and thus she focused only on seeing and enjoying the pictures displayed in the frame but did not understand what the auras were or that wellbeing information was sent to her son. The elderly was re-trained at $T_{1}$. She did not perceive her privacy was invaded and did not mind sharing the information with her children.

The elderly found it pleasing to see photos of her family on the picture frame and that his child was informed about changes in patterns and called when she had bad nights of sleep. Even though she could see his presence information and both could exchange affective messages, she still saw fewer benefits from the system for herself. Although she enjoyed seeing the emotions and also used them more at the beginning, like her son, she would have preferred to have more video and voice communication.

\section{Third generation of Aurama}

The first two versions of the Aurama system led to new insights and use cases for adopting such systems. Furthermore, a study was conducted with care providers from care organizations to understand their views of Aurama and what role it could play in their activities. Lastly, several new technology improvements were achieved that led to several changes in sensing and the design of new use cases based on the lessons from the earlier trials and the study with care providers.

\subsection{Involvement of care providers}

The preceding user studies discussed above have revealed that there is a need for the children to have further confidence regarding the information captured of the elderly, by either having a professional carer endorsing the information and/or "looking over the shoulder" of the system. This motivated an interview study involving 15 workers of Dutch and Belgian long-term care facilities including nurses, care planners, and facility managers. These were mainly semi-structured interviews with the aim of understanding the views of care organizations regarding the Aurama awareness system and how it could relate to their activities. These interviews were conducted in groups of two to six workers lasting between one and three hours.

The aim of a care facility is to deliver care services to its customers (elderly people) including: helping with activities of daily living such as dressing, washing, meals delivery, cleaning, etc.; providing prescribed medications on time; measuring vital signs (e.g., blood intake, pressure, weight); responding to emergency situations (skilled nurses and specialists are usually hired by the care facility); organizing social activities and social talks; evaluating care plans (twice a year per client); and advising to lower or increase the level of care (typically referred to geriatric support).

Care facilities continuously seek for strategies to reduce their costs and making their care process as efficient as possible. For example, by splitting social services from the healthcare ones and hiring people with lower education for social talks with the elderly; cooperating with external service companies for catering and cleaning; calling skilled nurses only when a real problem occurs; and by using very limited offthe-shelf technology. In fact, regarding the latter, a recent report from the Dutch Healthcare Inspection agency ('IGZ: Inspectie voor de Gezondheidszorg') 


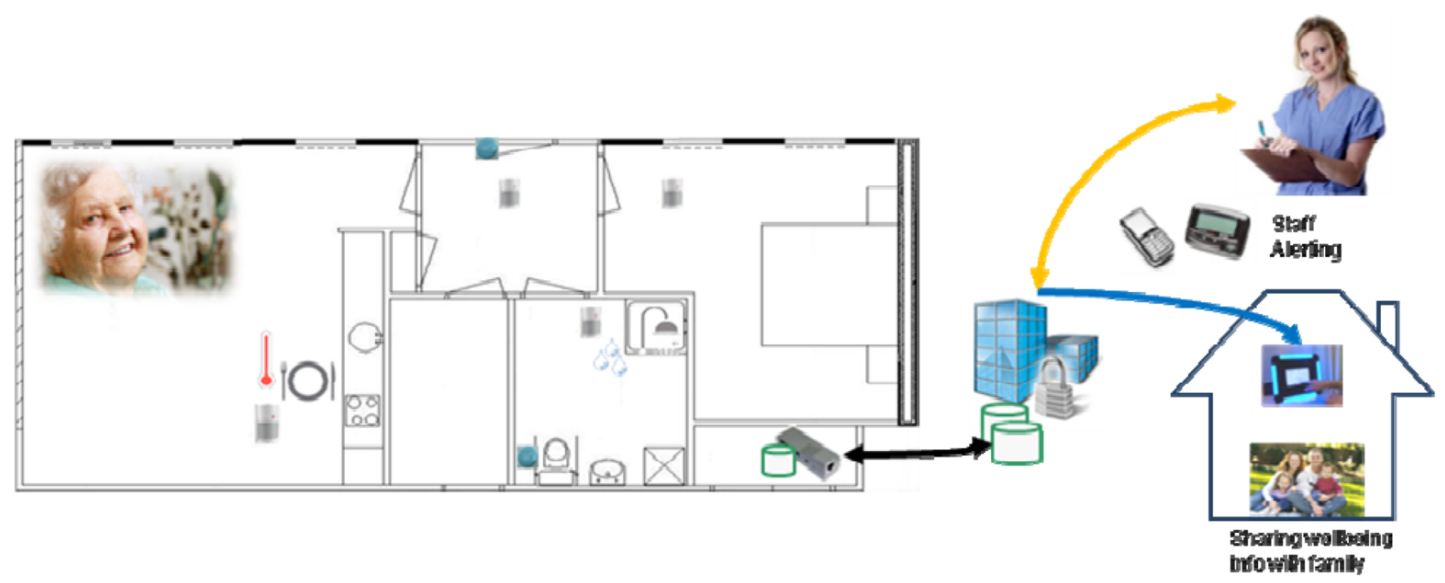

Fig. 5. Third generation of the Aurama awareness system: towards less obtrusive sensing and involving formal care providers.

[22], shows that the use of technology in the longterm care facilities in the Netherlands is still very limited. They are mostly around emergency response systems (typically a button worn by the elder which can be pressed in an emergency situation, or an 'all ok' button placed in the homes of an elder which they need to press every single morning to reassure the care providers that everything is fine with them. According to care providers, it happens often that elderly press the emergency button to trigger some social talk with a nurse who rushes to call in or to visit the elder. Furthermore, in one of the care organizations, over $20 \%$ of elderly do not press the 'all ok' button in the mornings causing unnecessary nurse visits and phone calls to the elder's apartment. Typically those elderly either do not like to press such a button, or do not understand the meaning of it, or simply forget to press it. Although it is clear that technologies can help in the care process and increase the safety of clients, they cannot replace care providers.

Care providers aim at increasing safety and quality of care and are more interested in emergency situations. With the increasing strain in the elderly care industry, they value the ability for elderly's children to become more proactive in the care of their aging parent. Thus, reducing the load from formal care providers, and have the children focus on long-term care and pattern changes. Furthermore, they foresee that Aurama could mitigate the isolation which is quite typical among elderly who live alone and at a distance from their children.

Two types of awareness information were identified as useful to embed in Aurama: (1) notification of alerts of potential emergency situations to care pro- viders and (2) early warning and preventive information based on long-term trends for elderly's children. The presentation of the awareness system should be different for each type of user. Care providers should be able to access the patterns and more detailed information via a web portal, while being notified of alerts via their current beeper or other mobile device. Children should get high-level information with some level of detail via the Aurama picture frame. Figure 5 shows an overview of the revised version of the Aurama awareness system.

\subsection{On-body vs. multimodal sensing}

To make the system less obtrusive for elderly, the presence detection mechanism using a wearable tag in a keychain was replaced with one based on sensors distributed in the physical environment but which do not require carrying a badge or other artefact. This is done by combining a switch sensor on the door for recognizing "door open/close" events and one motion sensor in the inner space near the door (e.g., a hall or a living room) for recognizing "movement" near the door. This sensor is placed at a certain distance from the door allowing the time difference between "open/close door" events and "movement in room" events to be discriminative. Such set-up allows counting time-stamped sequences and transition of events. A transition is a change of state of a sensor (e.g., from "no movement" to "movement") and a timeout is a specified period of time that when elapsed it could conclude a particular situation like "person has left the apartment": 'in-home movement' $\rightarrow$ 'door opened' $\rightarrow$ 'door closed' $\rightarrow$ specified timeout. This multi-modal and distributed sensor set-up 


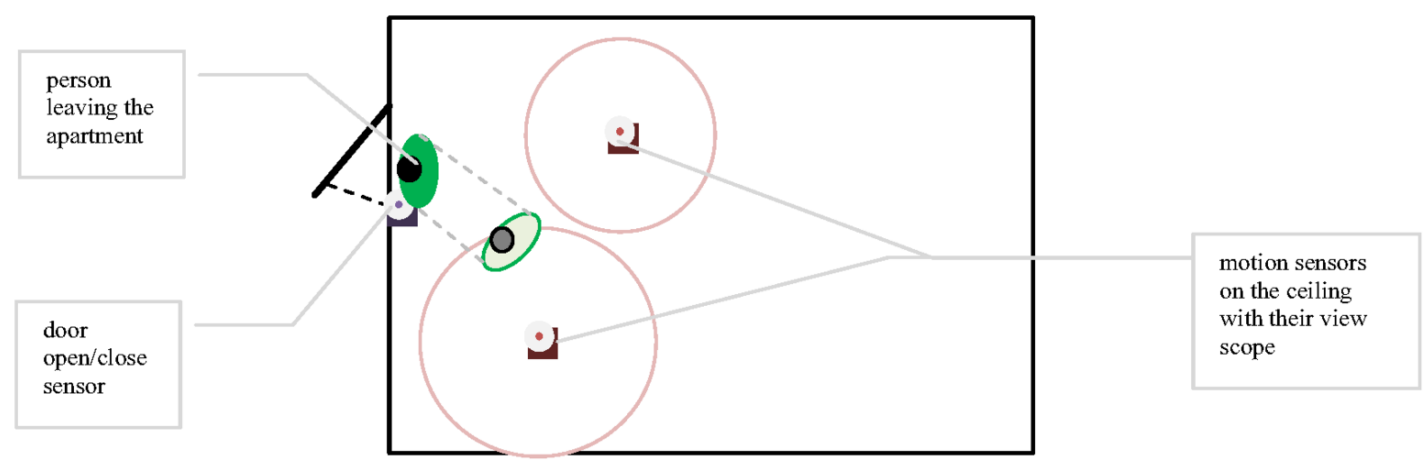

Fig. 6. Detecting home presence using distributed multimodal sensors.
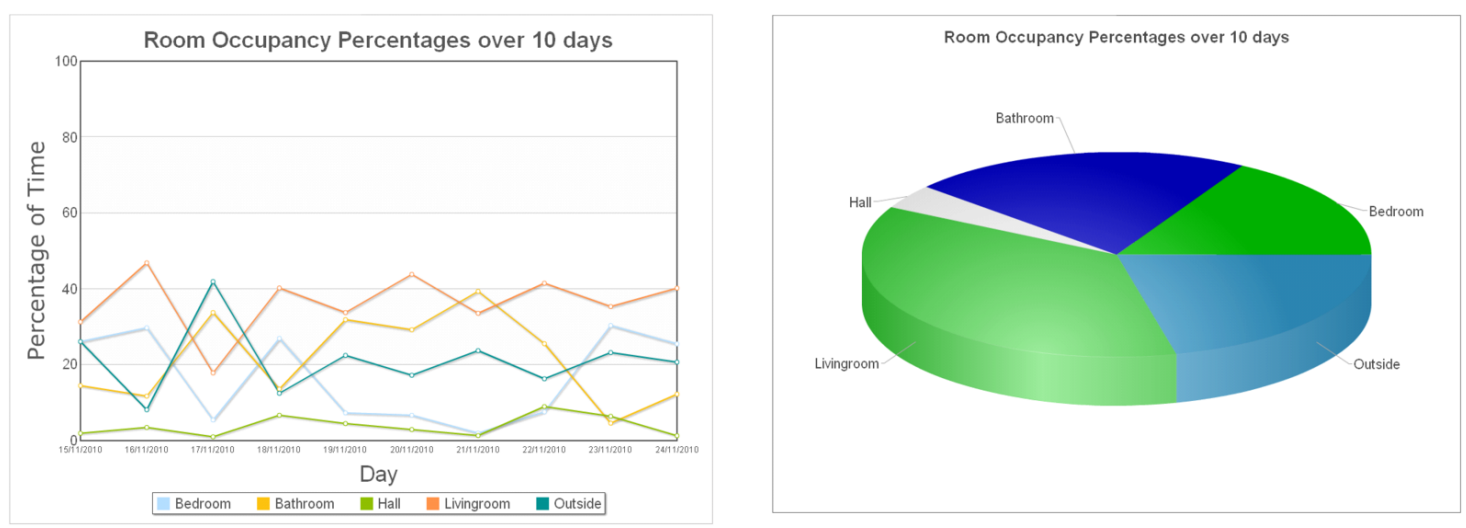

Fig. 7. Room occupancy was added to the third generation of the Aurama awareness system showing the difference between each space at home over time (left) and average room occupancy (right).

allows discriminating the following: a person opening the door and closing it but not leaving, a person moving near the door but not leaving, a person opening the door and closing it but not entering the apartment. Figure 6 shows a diagram of the new presence detection mechanism.

\subsection{Revised and new use cases}

It was clear from the initial studies that the load sensors under the bed were not as unobtrusive as had been anticipated. Thus, similarly to presence detection, sleep patterns detection using load cells was replaced with distributed sensors in the bedroom that capture bed activity and are much easy to install: a motion sensor placed near the bed to capture bed activity and a second motion sensor placed near the door of the bedroom. This combination allows the system to know whether the person enters or leaves the bedroom.
Even though weight is an important wellbeing parameter for elderly, the added value did not justify the burden of installing the load sensors under the bed and the ensuing inconvenience of having them there, so they were removed.

While presence detection and sleep patterns were revised, several new use cases were defined based on the interviews with elderly's children. For example, children are very interested in eating patterns and how active their parent is. Detection of these is done using a minimum set of unobtrusive motion sensors distributed in different areas at home, such as: expanding presence detection to room occupancy (i.e., the amount of time spent in each area at home, and identifying patterns of these; Fig. 7 shows a sample chart showing the average amount of time per area at home over a period of time), and patterns of kitchen visits. In addition to these, it would be interesting to explore patterns of bathroom visits and falls in the bathroom (since most falls of elderly happen in the bathroom). 
The revised presence and sleep patterns detection, bathroom visit trends, and room occupancy, have been implemented and future work will involve deploying the new Aurama system into the field.

\subsection{A back-end system}

A back-end system (as shown in Fig. 6) was added and placed remotely from the elderly's or child's home to have full remote access for potential adjustments overcoming issues with closed ports and firewalls encountered during previous field trials. The back-end system also allows running more computationally intensive algorithms to reason upon longterm trends to automatically identify changes in patterns. The back-end system offers as well a more controlled environment for data security, data archiving and load balancing. This architecture enables front-end devices like sensors, home hubs, and picture frames to be more "light-weight" and cheap.

\section{Implications for ambient intelligent systems}

This paper summarizes several user studies that have informed the iterative design of Aurama, and that helped evaluate the emerging user experience and the role this device can play for the elderly, their children and professional care givers. The trial deployments of different versions of Aurama support several conclusions regarding the role awareness systems can play for connecting elderly living independently and their adult children.

Pre and post calamity. Our participants considered the function of the system more appropriate for frailer elderly than those in our studies. This is consistent with Morris et al. [5], who derived a model of actual (decline) vs. perceived functioning (denial) of elderly, as seen in Fig. 8. Our data helps elaborate the awareness needs pertaining to different stages of the model. It is only after an event (e.g., a fall or hospitalization) that target users would perceive an awareness system to be useful; in this context the awareness of wellbeing becomes the primary goal. On the other hand, for those elderly who have not experienced such events, the primary goal of a system should be to provide new ways of communicating (e.g., easy text messages, video chat, or affective messages), and awareness of wellbeing to emerge as a by-product or a secondary benefit of using a system like Aurama.

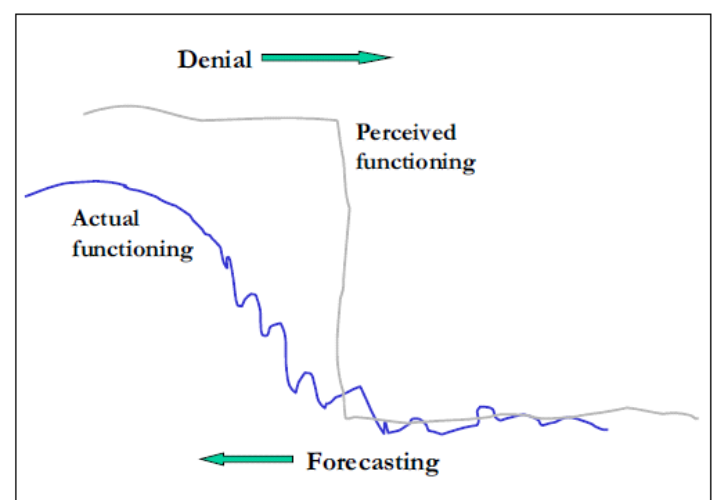

Fig. 8. Model of actual (decline) vs. perceived (denial) functioning of elderly from Morris et al. [5].

Awareness of what and for whom? Children want to be aware of changes they cannot grasp easily, looking at long-term trends to intervene on time before an event occurs. They do not want to receive detailed information on mundane daily activities of their parents that have little affective or functional value; rather they wish to have just enough information to detect early and easily changes relevant to the wellbeing of their parent that can trigger them to call and find out more or even to intervene and help. The preferred purpose of an awareness system is to trigger more contacts rather than replacing them.

Both elders and their children prefer that physiological measurements (e.g., blood pressure, glucose levels) be sent to a doctor. The third generation of the Aurama system completed the care service by including formal care providers. Notifications of potential emergency situations such as potential falls or leaving home at abnormal hours will be sent to care providers, while still having access to the same longterm trend information that the children have. Furthermore, the content and user interface has been tailored for each audience, e.g. ambient displays with high level awareness information for informal care givers and web portals with detailed awareness information for formal care providers. This involvement of care professionals was aimed at providing more confidence for target users and at increasing the acceptance of such systems.

Positive and negative news. Typically, people do not want to share negative news. In the field trials, both parties refrained from sending the cry emotion preferring to not explicitly provide awareness of negative feelings. However, when the system assessed bad nights of sleep and informed the family, it 
was perceived positively, given the reactions that they provoked. Elderly find it nice that children care for them and their family appreciate being involved in their wellbeing.

Intertwining implicit with explicit awareness of wellbeing. While implicit awareness of presence and wellbeing can provide peace of mind, its combination with the explicit exchange of affective awareness can further foster reassurance and connectedness. We have seen the value of this in the extensive field trials.

Allow for 'symmetric sharing'. During the preliminary trials, the system was mainly one-way flow of awareness information (from elderly to child). This gave a sense of a monitoring 'big brother' system. Having a two-way flow of awareness is essential for acceptance. Although awareness needs may be different between elderly and their children, there should be some symmetry on sharing awareness information.

Multimodal sensing. Field trials have clearly shown the benefits for relying on multiple and diverse sensors for drawing conclusions on a particular status or event. Presence, for example, was initially relied on a single active RF tag, placed in a keychain, but showed how participants would not always carry this with them and thus triggering false alarms. Using a non minimal set of sensors of different modalities distributed in the environment at strategic locations, it is possible to unobtrusively detect and reliably draw conclusions regarding the context of the elder, like their home presence status.

\section{Lessons learned from evaluating an awareness system in situ}

The experience in conducting long-term field trials with the Aurama awareness system has helped outline certain lessons for deploying such systems in situ:

Qualitiative versus Quantitative methods. The research field has paid a lot of attention to developing quantitative methods for evaluating the affective benefits and costs that accompany the use of systems like Aurama for informal communication with friends and family. Examples include the ABC questionnaire [24], the social connectedness questionnaire [23], to name a few. In cases where a complex and experimental installation is being evaluated in the wild', like in this article, such questionnaires have limited applicability. It is difficult to have large enough samples that would help identify statistically significant effects of introducing such a system into daily life. Testing for such long periods and in widely differing social and physical contexts, means that it would be impossible to attribute effects found to system use only: too many confounding variables prevent us from doing so. In this case, questionnaires can be used to provide some indicative quantification only and as interview guides for qualitative data collection.

Diary methods. While the results of the trials are predominantly based on qualitative feedback, the information gained could be even richer if such feedback would be probed throughout the study rather than at a few selected moments as we did in this study. Collecting data close to the moment of a particular experience and in the context where this experience concerns underpins the use of diary methods [28] or experience sampling methods, see for example [26] where a variant of experience sampling was used to study awareness needs between family members. However, such methods requiring regular reporting can put an excessive workload on participants also requiring them to use an application (the diary or experience sampling application) that can be more complex and more privacy threatening than the awareness system that is being evaluated.

To address this limitation, one could choose to have interventions during the study period, triggered by the sensed data. For example, if the elder has not slept well a particular night, at least following the indications obtained from the awareness system, this can be used by the researcher to trigger a telephone interview to both parent and child, or to trigger an interview through some other appropriate means (e.g., face to face for the elder, or instant messaging for the young adult). Such interventions can provide much richer data than an interview post-hoc at the end of a study phase, with the obvious downside of influencing the experience studied. While giving up aspirations to study experiences 'as they are' such an intervention driven approach can potentially provide more insight and more directed feedback regarding system behavior.

Deploying in phases. Unexpected technical challenges influence the feedback of the system. Having a research prototype deployed in the field makes it hard to avoid glitches that will require either temporarily (typically a couple of days) stopping the sys- 
tem operation while trying to fix the problem, or having to visit the participants home more than the planned and agreed upon number of times (sometimes several times a week), to either tweak the system and debug in situ. Field deployments of this kind can benefit by systematically planning the following three phases, and doing 'gating' to control transitions from one phase to the next: a) install sensors and gather data for some time till the system stabilizes and there is confidence regarding the collection of baseline data, b) the adaptive phase where algorithms and sensors are adjusted to deliver the required level of functionality and service in the installation context, and c) the deployment phase where the participants use and experience the system. This stepwise deployment approach was adopted for evaluating the second and third generations of the Aurama system, resulting in a more predictable and less burdensome deployment than for the first generation, where this phasing and checks had not been foreseen.

Aesthetically blending into the environment. One of the main aims of the system is to be as unobtrusive as possible. However, the prototype system caused some disturbances to participants that at times distracted them from assessing the value of the actual concept. Some examples were the load cells causing their bed to be a few centimeters higher, not being able to clean or vacuum properly under the bed due to the set up, or in some environments the light aura on the frame was too bright. In short, aesthetics play a role too not only for products but for the smooth execution of a field deployment. For example, participants found the frame too big for their living rooms and were reluctant to place it at a place congruent with the design motivation of the research team. Although building a prototype system that is close to the desired end product is not always easy, it is important to mitigate any disturbances as much as possible and make it blend into their deployment environment in order to allow researchers to gauge the resulting user experience and to obtain relevant feedback regarding the role and value of such systems.

\section{Conclusions}

This paper has presented the design and evaluation of Aurama an awareness system to support awareness of elderly living alone. The system went through several iterations of development and testing. The length of field deployments was gradually increased from two-week long trials, to two extensive four-to-six month's long field trials.

These trials have confirmed and increased our confidence and understanding regarding the benefit of such applications. Most important, awareness systems are not seen as a new "Panopticon" that lets one monitor the mundane activities of the other unobserved. Rather they are put forward as a compliment to other means of communication and social contact, they need to be designed to be embedded in such daily informal and playful communication activities, while at the same time providing an opportunity to uncover latent problems and long-term trends, bringing objective measurement to complement personal impressions and, where necessary, putting professional experts in the loop.

The third generation of Aurama has been implemented and described in this article. It includes care providers and makes the system less obtrusive covering a wider set of use cases based on the lessons learned from the initial studies and trials. Future work aims at deploying and further evaluating the new system in longer-term field studies.

\section{References}

[1] S. Consolvo, P. Roessler and B. Shelton, The CareNet display: Lessons learned from an in home evaluation of an ambient display, in: Proceedings of Ubicomp, 2004.

[2] G. Metaxas, B. Metin, J. Schneider, P. Markopoulos and B. de Ruyter, Diarist: Aging in place with semantically enriched narratives, in: Proceedings of Interact, 2007.

[3] J. Rowan and E. Mynatt, digital family portrait field trial: Support for aging in place, in: Proceedings of CHI 2005 ACM Press, 2005, pp. 521-530.

[4] E. Aarts, H. Harwig and M. Schuurmans, Ambient intelligence, in: The Invisible Future, J. Denning, ed., McGrawHill, New York, 2001, pp. 235-250.

[5] M. Morris, J. Lundell, E. Dishman and B. Needham, New perspectives on ubiquitous computing from ethnographic study of elders with cognitive decline, in: Proceedings of Ubicomp, Springer, 2003, pp. 227-242.

[6] J. Van Baren, W. IJsselsteijn, P. Markopoulos, N. Romero and B. De Ruyter, Measuring affective benefits and costs of awareness systems supporting intimate social networks, in: CTIT Workshop Proceedings Series (2), Proceedings of Social Intelligence Design, A. Nijholt and T. Nishida, eds, 2004.

[7] P. Dadlani, A. Sinitsyn, W. Fontijn and P. Markopoulos, $A u$ rama: Caregiver Awareness for Living Independently with an Augmented Picture Frame Display, Artificial Intelligence \& Society, Springer, 2010.

[8] R.F Baumeister and M.R. Leary, The need to belong: Desire for interpersonal attachments as a fundamental human motivation, Psychology Bulletin 117(3) (1995), 497-529.

[9] E. Aarts and S. Marzano, eds, The New Everyday: Visions of Ambient Intelligence, 010 Publishing, Rotterdam, 2003. 
[10] P. Dadlani, P. Markopoulos and E. Aarts, Intertwining implicit and explicit awareness of wellbeing to support peace of mind and connectedness, in: Proceedings of $3^{\text {rd }}$ European Conference of Ambient Intelligence, 2009, pp. 153-158.

[11] E. Mynatt, J. Rowan, S. Craighill and A. Jacobs, Digital family portraits: Supporting peace of mind for extended family members, in: Proceedings of CHI 2001, ACM Press, 2001.

[12] QuietCare: http://www.quietcaresystems.com/.

[13] HealthSense: http://www.healthsense.com/.

[14] WellAware: http://www.wellawaresystems.com/.

[15] J. Kelley, An iterative design methodology for userfriendly natural language office information applications, Proceedings of ACM Transactions Office Information Systems 2(1) (1984).

[16] J.V. Khan and P. Markopoulos, Busy families' awareness needs, International Journal of Human-Computer Studies, Springer 67(2) (2009), 139-153.

[17] B.A.T. Brown, A.S. Taylor, S. Izadi, A. Sellen, J. Kaye and R. Eardley, Locating family values: A field trial of the whereabouts clock, in: Ubicomp, J. Krumm, G.D. Abowd, A. Seneviratne and T. Strang, eds, LNCS, Vol. 4717, Springer, pp. 354-371.

[18] Z. Pousman and J. Stasko, A taxonomy of ambient information systems: Four patterns of design, in: Proceedings of the Working Conference on Advanced Visual Interfaces, ACM Press, 2006, pp. 67-74.

[19] J. Short, E. Williams and B. Christie, The Social Psychology of Telecommunications, Wiley, 1976.

[20] J. Hollan and S. Stornetta, Beyond being there, in: Proceedings of the SIGCHI Conference on Human Factors in Computing Systems (CHI'92), P. Bauersfeld, J. Bennett and G. Lynch, eds, ACM, New York, NY, USA, pp. 119-125,
DOI=10.1145/142750.142769, http://doi.acm.org/10.1145/ 142750.142769

[21] Federal Interagency Forum on Aging-Related Statistics, Older Americans 2008: Key Indicators of Well-Being, 2008.

[22] Toepassing van domotica in de zorg moet zorgvuldiger, Rapport van Inspectie voor de Gezonheidszorg, oktober 2009.

[23] D. Van Bel, K. Smolders, W. IJsselsteijn and Y. de Kort, Social connectedness: Concept and measurement, in: Proceedings of Intelligent Environments, IOS, 2009.

[24] J. Van Baren, W. IJsselsteijn, N. Romero, P. Markopoulos and B. De Ruyter, Affective benefits in communication: The development and field-testing of a new questionnaire measure, in: Proceedings of PRESENCE, 2003.

[25] E. McAuley and T. Duncan, Psychometric properties of the intrinsic motivation inventory in a competitive sport setting. A conformation factor analysis, Research Quart. for Exercise \& Sport 60(1) (1989), 48-58.

[26] V. Khan, P. Markopoulos, B. Eggen, W. IJsselsteijn and B. De Ruyter, Reconexp: A way to reduce the data loss of the experiencing sampling method, in: Proceedings of MobileHCI 2008, ACM, New York, NY, 68, 2008, pp. 471-476.

[27] P. Markopoulos, B. De Ruyter and W. Mackay, eds, Awareness Systems: Advances in Theory, Methodology and Design, Human-Computer Interaction Series, 2009, Approx. 485 p., 98 illus., Hardcover, ISBN: 978-1-84882-476-8, DOI 10.1007/978-1-84882-477-5.

[28] J. Rieman, The diary study: A workplace-oriented research tool to guide laboratory efforts, in: Proceedings of the INTERACT'93 and CHI'93 Conference on Human Factors in Computing Systems, ACM Press, New York, NY, USA, pp. 321-326, DOI=10.1145/169059.169255, http://doi.acm.org $10.1145 / 169059.169255$. 